\title{
SIGN PATTERNS THAT ALLOW A POSITIVE OR NONNEGATIVE LEFT INVERSE*
}

\author{
IN-JAE KIM ${ }^{\dagger}$, D. D. OLESKY ${ }^{\ddagger}$, B. L. SHADER ${ }^{\S}$, AND P. VAN DEN DRIESSCHE
}

\begin{abstract}
An $m$ by $n$ sign pattern $\mathcal{S}$ is an $m$ by $n$ matrix with entries in $\{+,-, 0\}$. Such a sign pattern allows a positive (resp., nonnegative) left inverse, provided that there exist an $m$ by $n$ matrix $A$ with the sign pattern $\mathcal{S}$ and an $n$ by $m$ matrix $B$ with only positive (resp., nonnegative) entries satisfying $B A=I_{n}$, where $I_{n}$ is the $n$ by $n$ identity matrix. For $m>n \geq 2$, a characterization of $m$ by $n$ sign patterns with no rows of zeros that allow a positive left inverse is given. This leads to a characterization of all $m$ by $n$ sign patterns with $m \geq n \geq 2$ that allow a positive left inverse, giving a generalization of the known result for the square case, which involves a related bipartite digraph. For $m \geq n, m$ by $n$ sign patterns with all entries in $\{+, 0\}$ and $m$ by 2 sign patterns with $m \geq 2$ that allow a nonnegative left inverse are characterized, and some necessary or sufficient conditions for a general $m$ by $n$ sign pattern to allow a nonnegative left inverse are presented.
\end{abstract}

Key words. bipartite digraph, nonnegative left inverse, positive left inverse, positive left nullvector, sign pattern, strong Hall

AMS subject classifications. 15A09, 15A48, 05C20, 05C50

DOI. $10.1137 / 060660916$

1. Introduction. An $m$ by $n$ sign pattern $\mathcal{S}=\left[s_{i j}\right]$ is an $m$ by $n$ matrix with entries in $\{+,-, 0\}$. If a sign pattern $\mathcal{S}$ has all entries in $\{+, 0\}$, then $\mathcal{S}$ is a nonnegative sign pattern. A subpattern of $\mathcal{S}$ is an $m$ by $n \operatorname{sign}$ pattern $\mathcal{U}=\left[u_{i j}\right]$ such that $u_{i j}=0$ whenever $s_{i j}=0$. If $\mathcal{U}$ is a subpattern of $\mathcal{S}$, then $\mathcal{S}$ is a superpattern of $\mathcal{U}$. The sign pattern class $Q(\mathcal{S})$ of an $m$ by $n$ sign pattern $\mathcal{S}$ is the set of $m$ by $n$ matrices $A=\left[a_{i j}\right]$ such that $\operatorname{sgn}\left(a_{i j}\right)=s_{i j}$ for all $i, j$. If $A \in Q(\mathcal{S})$, then $A$ is a realization of $\mathcal{S}$.

Let $A=\left[a_{i j}\right]$ be an $m$ by $n$ matrix. If each entry of $A$ is positive (resp., nonnegative), then $A$ is positive (resp., nonnegative), written $A>0$ (resp., $A \geq 0$ ). A left inverse of an $m$ by $n$ matrix $A$ is an $n$ by $m$ matrix $B$ such that $B A=I_{n}$, where $I_{n}$ denotes the $n$ by $n$ identity matrix. If $B>0$, then $B$ is a positive left inverse (abbreviated as $P L I$ ) of $A$. If $B \geq 0$, then $B$ is a nonnegative left inverse (abbreviated as $N L I)$ of $A$. In general, neither a PLI nor an NLI of $A$ is unique. It is easily verified that $A$ has a left inverse if and only if $\operatorname{rank} A=n$; thus, if $A$ has a left inverse, then necessarily $m \geq n$. An $m$ by $n$ sign pattern $\mathcal{S}$ allows a positive (resp., nonnegative) left inverse, provided there exist $A \in Q(\mathcal{S})$ and a matrix $B>0$ (resp., $B \geq 0$ ) such that $B A=I_{n}$. Note that if $P_{1}$ and $P_{2}$ are permutation matrices, then $\mathcal{S}$ allows a PLI (resp., an NLI) if and only if $P_{1} \mathcal{S} P_{2}$ allows a PLI (resp., an NLI).

*Received by the editors May 25, 2006; accepted for publication (in revised form) by R. Bhatia October 5, 2006; published electronically April 20, 2007.

http://www.siam.org/journals/simax/29-2/66091.html

${ }^{\dagger}$ Department of Mathematics and Statistics, Minnesota State University, Mankato, MN 56001 (in-jae.kim@mnsu.edu). The research of this author was done while he was a postdoctoral fellow at the University of Victoria.

${ }^{\ddagger}$ Department of Computer Science, University of Victoria, P.O. Box 3055, Victoria, BC, Canada V8W 3P6 (dolesky@cs.uvic.ca). The research of this author was supported in part by an NSERC Discovery Grant.

$\S$ Department of Mathematics, University of Wyoming, Laramie, WY 82071 (bshader@uwyo.edu).

๑ Department of Mathematics and Statistics, University of Victoria, P.O. Box 3045, Victoria, BC, Canada V8W 3P4 (pvdd@math.uvic.ca). The research of this author was supported in part by an NSERC Discovery Grant. 
A motivation for studying PLIs and NLIs comes from determining the qualitative behavior of solutions of $A^{T} x=b$ with $A$ an $m$ by $n$ matrix; see, for example, [2, Chapter 1] and [5] for applications in economics. Specifically, $A$ has a PLI (resp., an NLI) if and only if for each $n$ by 1 nonzero vector $b \geq 0$ there exists an $m$ by 1 vector $x>0$ (resp., $x \geq 0$ ) satisfying $A^{T} x=b$ or equivalently $x^{T} A=b^{T}$; see Proposition 4.1 for a proof.

Square sign patterns with entries in $\{+,-\}$ that allow a positive (left) inverse are characterized in [6], and this characterization is extended to arbitrary square sign patterns in [4]. These results are summarized in [2, section 9.2]. In section 2, we characterize nonsquare sign patterns that allow a PLI, and combine the square and nonsquare characterizations. In section 3, we discuss sign patterns that allow an NLI. More specifically, we characterize nonnegative sign patterns and $m$ by 2 sign patterns with $m \geq 2$ that allow an NLI, and present some necessary or sufficient conditions for general $m$ by $n$ sign patterns with $m \geq n$ to allow an NLI. We conclude with some remarks in section 4 .

2. Positive left inverses. We begin this section with a necessary and sufficient condition for a column sign pattern to allow a PLI or an NLI.

Proposition 2.1. Let $\mathcal{S}=\left(s_{1}, s_{2}, \ldots, s_{m}\right)^{T}$ be an $m$ by 1 sign pattern. Then the following are equivalent:

(i) $\mathcal{S}$ has a + entry.

(ii) $\mathcal{S}$ allows a PLI.

(iii) $\mathcal{S}$ allows an $N L I$.

Proof. Suppose there is an index $i \in\{1,2, \ldots, m\}$ with $s_{i}=+$. For $j \in$ $\{1, \ldots, m\}$, set

$$
a_{j}= \begin{cases}1 & \text { if } j \neq i \text { and } s_{j}=+, \\ -1 & \text { if } j \neq i \text { and } s_{j}=-, \\ 0 & \text { if } j \neq i \text { and } s_{j}=0, \\ 1+\sum_{k \neq i}\left|a_{k}\right| & \text { if } j=i .\end{cases}
$$

Then $A=\left(a_{1}, \ldots, a_{m}\right)^{T} \in Q(\mathcal{S})$, and $(1,1, \ldots, 1) A=1+\sum_{k \neq i}\left(\left|a_{k}\right|+a_{k}\right)=c>0$. This implies that $\frac{1}{c}(1,1, \ldots, 1)$ is a PLI of $A$. Thus, $\mathcal{S}$ allows a PLI.

It is clear that (ii) implies (iii). Next, suppose that the sign pattern $\mathcal{S}$ allows an NLI. Then there exist $A=\left(a_{1}, \ldots, a_{m}\right)^{T} \in Q(\mathcal{S})$ and $B=\left(b_{1}, \ldots, b_{m}\right) \geq 0$ such that $B A=1$, i.e., $\sum_{j=1}^{m} b_{j} a_{j}=1>0$. This implies that there exists an $i$ with $b_{i} a_{i}>0$. Since $b_{i} \geq 0$, it follows that $b_{i}>0$; hence $a_{i}>0$ and thus $s_{i}=+$.

We now consider $m \geq n \geq 2$. The following two lemmas give necessary conditions for a sign pattern to allow a PLI.

LEMma 2.2. Let $\mathcal{S}$ be an $m$ by $n$ sign pattern with $n \geq 2$. If $\mathcal{S}$ allows a PLI, then each column of $\mathcal{S}$ has $a+$ and $a-$ entry.

Proof. Suppose that there exist $A \in Q(\mathcal{S})$ and an $n$ by $m$ positive matrix $B$ such that $B A=I_{n}$. Let $i \in\{1,2, \ldots, n\}$. Since the $(i, i)$-entry of $B A$ is 1 and each entry of $B$ is positive, it follows that some entry in column $i$ of $A$ is positive. Hence, column $i$ of $\mathcal{S}$ has a + entry.

Since $n \geq 2$, there exists $j \in\{1, \ldots, n\}$ with $j \neq i$. The $(j, i)$-entry of $B A$ is 0 , so since $B>0$ and at least one entry of column $i$ of $A$ is positive, it follows that at least one entry of column $i$ of $A$ must be negative. Thus, column $i$ of $\mathcal{S}$ has a entry.

An $m$ by $n$ sign pattern $\mathcal{S}$ with $n \geq 2$ is strong Hall, provided that for every nonempty proper subset $\gamma$ of $\{1,2, \ldots, n\}$ the submatrix of $\mathcal{S}$ consisting of the columns 
indexed by $\gamma$ has nonzero entries in at least $|\gamma|+1$ rows (see [3]). Note that if $\mathcal{S}$ is strong Hall, then necessarily $m \geq n$. Also, for $m \geq n, \mathcal{S}$ is not strong Hall if and only if there exist permutation matrices $P_{1}$ and $P_{2}$ such that

$$
P_{1} \mathcal{S} P_{2}=\left[\begin{array}{cc}
\mathcal{S}_{11} & \mathcal{S}_{12} \\
O & \mathcal{S}_{22}
\end{array}\right]
$$

where $\mathcal{S}_{11}$ is a $k$ by $\ell$ sign pattern for some integers $k, \ell$ with $n>\ell \geq 1$ and $k \leq \ell$.

LEMma 2.3. Let $\mathcal{S}$ be an $m$ by $n$ sign pattern with $n \geq 2$. If $\mathcal{S}$ allows a PLI, then $\mathcal{S}$ is strong Hall.

Proof. To prove the contrapositive, assume that $\mathcal{S}$ is not strong Hall. If $m<n$, then it is clear that $\mathcal{S}$ does not allow a PLI. Otherwise, without loss of generality, we may assume that $\mathcal{S}$ has the form (2.1). If $k<\ell$, then the first $\ell$ columns of each realization of $\mathcal{S}$ are linearly dependent, and hence $\mathcal{S}$ does not allow a PLI.

Otherwise, $k=\ell<n$. Suppose that there exists a matrix $A=\left[\begin{array}{cc}A_{11} & A_{12} \\ O & A_{22}\end{array}\right] \in Q(\mathcal{S})$ with a left inverse $B=\left[\begin{array}{ll}B_{11} & B_{12} \\ B_{21} & B_{22}\end{array}\right]$, where $B_{11}$ is an $\ell$ by $\ell$ matrix. Clearly, the $\ell$ by $\ell$ matrix $A_{11}$ is invertible, and by $B A=I_{n}$, it follows that $\left[\begin{array}{ll}B_{21} & B_{22}\end{array}\right]\left[\begin{array}{c}A_{11} \\ O\end{array}\right]=O$. Thus, $B_{21} A_{11}=O$, and since $A_{11}$ is invertible, the $(n-\ell)$ by $\ell$ matrix $B_{21}=O$. Since $n-\ell \geq 1$ and $\ell \geq 1$, every left inverse of a matrix in $Q(\mathcal{S})$ has a zero entry, and hence $\mathcal{S}$ does not allow a PLI.

Note that if $\mathcal{S}$ is a square sign pattern of order $n \geq 2$, then $\mathcal{S}$ is strong Hall if and only if $\mathcal{S}$ is fully indecomposable (see [3]), and $\mathcal{S}$ allows a PLI if and only if $\mathcal{S}$ allows a positive inverse. The next theorem, first proved in [4], provides a characterization of square sign patterns that allow a positive inverse. In order to recall this characterization, we use the following definition as in [1] and [2]. Let $\mathcal{S}=\left[s_{i j}\right]$ be an $m$ by $n$ sign pattern. The bipartite digraph $D(\mathcal{S})$ of $\mathcal{S}$ is the digraph with row vertices $u_{1}, \ldots, u_{m}$, column vertices $v_{1}, \ldots, v_{n}$, an $\operatorname{arc} u_{i} \rightarrow v_{j}$ if $s_{i j}=+$, and an $\operatorname{arc} v_{j} \rightarrow u_{i}$ if $s_{i j}=-$. Note that there exists at most one arc between $u_{i}$ and $v_{j}$.

Theorem 2.4 (see [2, Theorem 9.2.1]). An $n$ by $n$ square sign pattern $\mathcal{S}$ with $n \geq 2$ allows a positive (left) inverse if and only if $\mathcal{S}$ is strong Hall and the bipartite digraph $D(\mathcal{S})$ of $\mathcal{S}$ is strongly connected.

Let $\mathcal{S}$ be an $m$ by $n$ sign pattern and let $D(\mathcal{S})$ be its bipartite digraph. A strong component of $D(\mathcal{S})$ is a maximal strongly connected subdigraph of $D(\mathcal{S})$. If $\alpha$ is a strong component of $D(\mathcal{S})$, then $|\alpha|$ denotes the number of vertices in $\alpha$.

Remark 2.5. Let $\alpha$ be a strong component of $D(\mathcal{S})$. Since $D(\mathcal{S})$ is a bipartite digraph with no cycles of length 2 , it follows that if $|\alpha| \geq 2$, then $\alpha$ has at least two row vertices and at least two column vertices.

Let $\alpha_{1}, \alpha_{2}, \ldots, \alpha_{t}$ be the strong components of $D(\mathcal{S})$. The condensed digraph $C D(\mathcal{S})$ of $\mathcal{S}$ has vertices $\alpha_{1}, \alpha_{2}, \ldots, \alpha_{t}$ and an $\operatorname{arc} \alpha_{i} \rightarrow \alpha_{j}$ if and only if $i \neq j$ and $D(\mathcal{S})$ has at least one arc from a vertex in $\alpha_{i}$ to a vertex in $\alpha_{j}$. A strong component $\alpha_{i}$ of $D(\mathcal{S})$ is a source if there is no arc coming into $\alpha_{i}$ in $C D(\mathcal{S})$ and there is at least one arc coming out of $\alpha_{i}$ in $C D(\mathcal{S}) ; \alpha_{i}$ is a sink if there is no arc coming out of $\alpha_{i}$ in $C D(\mathcal{S})$ and there is at least one arc coming into $\alpha_{i}$ in $C D(\mathcal{S})$; and $\alpha_{i}$ is isolated if there are no arcs coming into or out of $\alpha_{i}$ in $C D(\mathcal{S})$.

LemMa 2.6. Let $\mathcal{S}$ be an $m$ by $n$ sign pattern which has $a+$ and a-entry in each column and no rows of zeros. Then the following hold for $D(\mathcal{S})$ :

(i) Each sink and source strong component of $D(\mathcal{S})$ has at least one row vertex.

(ii) Each isolated strong component has at least two row vertices.

Proof. (i) Let $\alpha$ be a sink or source strong component. If $|\alpha|=1$, then since each column of $\mathcal{S}$ has a + and a - entry, it follows that no sink or source strong component 
consists of exactly one column vertex. Hence, $\alpha$ is a row vertex. If $|\alpha| \geq 2$, then Remark 2.5 implies that $\alpha$ has at least one row vertex.

(ii) By the assumptions on the rows and columns of $\mathcal{S}$, there is no isolated strong component with exactly one vertex. Hence, by Remark 2.5, each isolated strong component has at least two row vertices.

Let $A$ be an $m$ by $n$ matrix with $m \geq n$. If there exists an $m$ by 1 vector $y>0$ satisfying $y^{T} A=0$, then $y^{T}$ is a positive left nullvector of $A$. The following theorem gives a characterization of nonsquare sign patterns with no rows of zeros that allow a PLI. Note that conditions for such a sign pattern to allow a PLI are weaker than those for square sign patterns (Theorem 2.4), although the bipartite digraph is used in our proof for a nonsquare sign pattern.

TheOREM 2.7. For $m>n \geq 2$, let $\mathcal{S}$ be an $m$ by $n$ sign pattern with no rows of zeros. Then the following are equivalent:

(i) There exists a matrix $A \in Q(\mathcal{S})$ with a PLI and a positive left nullvector.

(ii) $\mathcal{S}$ allows a PLI.

(iii) Each column of $\mathcal{S}$ has a + and a - entry, and $\mathcal{S}$ is strong Hall.

Proof. Clearly, (i) implies (ii). By Lemmas 2.2 and 2.3, (ii) implies (iii).

To prove that (iii) implies (i), assume that $\mathcal{S}$ is strong Hall and that $\mathcal{S}$ has a + and a - entry in each column. We claim that it suffices to show that there exists an $m$ by $(m-n)$ sign pattern $\mathcal{C}$ so that the $m$ by $m \operatorname{sign}$ pattern $[\mathcal{S} \mid \mathcal{C}]$ allows a positive (left) inverse. To prove this claim, suppose there exists an $m$ by $m$ matrix $[A \mid C] \in Q([\mathcal{S} \mid \mathcal{C}])$ with a positive (left) inverse $\left[\begin{array}{l}B_{1} \\ B_{2}\end{array}\right]$ where $B_{1}$ is an $n$ by $m$ positive matrix and $B_{2}$ is an $(m-n)$ by $m$ positive matrix. Then $B_{1} A=I_{n}$ and hence $B_{1}$ is a PLI of $A$, implying that $\mathcal{S}$ allows a PLI. In addition, since $B_{2} A=O$ and $B_{2}$ has at least one positive row, $A$ has a positive left nullvector. Therefore, by Theorem 2.4, it suffices to find an $m$ by $(m-n) \operatorname{sign}$ pattern $\mathcal{C}$ such that the $m$ by $m$ sign pattern $[\mathcal{S} \mid \mathcal{C}]$ is strong Hall and its bipartite digraph $D([\mathcal{S} \mid \mathcal{C}])$ is strongly connected.

Consider the bipartite digraph $D(\mathcal{S})$ of $\mathcal{S}$. Let $\alpha_{1}, \alpha_{2}, \ldots, \alpha_{t}$ be its strong components, where $\alpha_{1}, \ldots, \alpha_{k}$ are sinks, $\alpha_{k+1}, \ldots, \alpha_{k+\ell}$ are sources, $\alpha_{k+\ell+1}, \ldots, \alpha_{k+\ell+r}$ are isolated, and $\alpha_{k+\ell+r+1}, \ldots, \alpha_{t}$ are neither sinks, sources, nor isolated strong components. By Lemma 2.6 (i), each sink and source strong component has a row vertex. Let $r_{i}$ be a fixed row vertex of $\alpha_{i}$ for each $i \in\{1, \ldots, k+\ell\}$. Also, by Lemma 2.6 (ii), each isolated strong component has at least two row vertices. Let $r_{i}^{+}, r_{i}^{-}$be distinct fixed row vertices of $\alpha_{i}$ for each $i \in\{k+\ell+1, \ldots, k+\ell+r\}$. Let $\mathcal{C}_{n+1}$ be the $m$ by 1 column sign pattern with nonzero $j$ th coordinate:

$$
\left\{\begin{aligned}
+ & \text { if } u_{j} \in\left\{r_{1}, \ldots, r_{k}\right\} \cup\left\{r_{k+\ell+1}^{-}, \ldots, r_{k+\ell+r}^{-}\right\} \\
- & \text {if } u_{j} \in\left\{r_{k+1}, \ldots, r_{k+\ell}\right\} \cup\left\{r_{k+\ell+1}^{+}, \ldots, r_{k+\ell+r}^{+}\right\} \\
+ & \text {otherwise. }
\end{aligned}\right.
$$

Then $D\left(\left[\mathcal{S} \mid \mathcal{C}_{n+1}\right]\right)$ is obtained from $D(\mathcal{S})$ by appending a new column vertex $c_{n+1}$, and $\operatorname{arcs} r_{j} \rightarrow c_{n+1}$ if $r_{j}$ is in a sink component; $c_{n+1} \rightarrow r_{j}$ if $r_{j}$ is in a source component; $r_{j}^{-} \rightarrow c_{n+1}$ and $c_{n+1} \rightarrow r_{j}^{+}$if $r_{j}^{-}$and $r_{j}^{+}$are in the same isolated component; as well as some additional arcs coming into vertex $c_{n+1}$.

To prove that $D\left(\left[\mathcal{S} \mid \mathcal{C}_{n+1}\right]\right)$ is strongly connected, we show that for each vertex $w$ of $D(\mathcal{S})$ there exists in $D\left(\left[\mathcal{S} \mid \mathcal{C}_{n+1}\right]\right)$ a walk from $c_{n+1}$ to $w$ and a walk from $w$ to $c_{n+1}$. Note that if $w$ is not in an isolated strong component of $D(\mathcal{S})$, then there is a walk from $w$ to a vertex in a sink strong component $\alpha_{i}$ of $D(\mathcal{S})(i \in\{1, \ldots, k\})$. Since $\alpha_{i}$ is strongly connected, this walk from $w$ can be extended to the fixed row vertex $r_{i}$ of $\alpha_{i}$. By $(2.2)$, there is an $\operatorname{arc} r_{i} \rightarrow c_{n+1}$ in $D\left(\left[\mathcal{S} \mid \mathcal{C}_{n+1}\right]\right)$. Hence, there is 
a walk from $w$ to $c_{n+1}$. Similarly, there is a walk from $c_{n+1}$ to $w$.

Next, suppose that $w$ is a vertex in an isolated strong component $\alpha_{i}$ in $D(\mathcal{S})$ $(i \in\{k+\ell+1, \ldots, k+\ell+r\})$. Since $\alpha_{i}$ is strongly connected, there is a walk from $w$ to the fixed row vertex $r_{i}^{-}$of $\alpha_{i}$. By (2.2), there are $\operatorname{arcs} r_{i}^{-} \rightarrow c_{n+1}$ and $c_{n+1} \rightarrow r_{i}^{+}$ in $D\left(\left[\mathcal{S} \mid \mathcal{C}_{n+1}\right]\right)$. Since $\alpha_{i}$ is strongly connected, there is a walk from $r_{i}^{+}$to $w$. Thus, there exist a walk from $w$ to $c_{n+1}$ and a walk from $c_{n+1}$ to $w$.

Finally, define $\mathcal{C}_{n+2}, \ldots, \mathcal{C}_{m}$ to be $m$ by 1 column sign patterns, each having no zeros, at least one + , and at least one - entry. Then it is easily verified that $D\left(\left[\mathcal{S}\left|\mathcal{C}_{n+1}\right| \ldots \mid \mathcal{C}_{m}\right]\right)$ is strongly connected. Since $\mathcal{S}$ is strong Hall and $\left[\mathcal{C}_{n+1}|\ldots| \mathcal{C}_{m}\right]$ has no zeros, it is clear that $\left[\mathcal{S}\left|\mathcal{C}_{n+1}\right| \ldots \mid \mathcal{C}_{m}\right]$ is strong Hall, completing the proof.

Example 2.8. Consider the 6 by 4 sign pattern

$$
\mathcal{S}=\left[\begin{array}{cccc}
+ & - & 0 & 0 \\
- & + & 0 & 0 \\
+ & - & 0 & 0 \\
0 & 0 & + & - \\
0 & 0 & - & + \\
0 & 0 & 0 & -
\end{array}\right]
$$

with
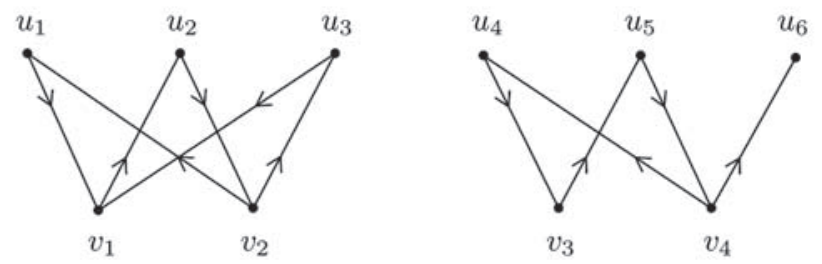

Each column of $\mathcal{S}$ has a + and a - entry, and $\mathcal{S}$ is strong Hall. Thus, by Theorem 2.7, $\mathcal{S}$ allows a PLI. However, $D(\mathcal{S})$ is not strongly connected, illustrating a distinction between the nonsquare and square cases (see Theorem 2.4). In fact, $D(\mathcal{S})$ has one sink strong component $\alpha_{1}$ that consists of vertex $u_{6}$, one source strong component $\alpha_{2}$ with vertices $u_{4}, u_{5}, v_{3}, v_{4}$, and one isolated strong component $\alpha_{3}$ with vertices $u_{1}, u_{2}, u_{3}, v_{1}, v_{2}$. Taking $r_{1}=u_{6}, r_{2}=u_{5}, r_{3}^{+}=u_{1}$, and $r_{3}^{-}=u_{2}$ in the proof of Theorem 2.7, it follows that

$$
\mathcal{C}_{5}=\left[\begin{array}{c}
- \\
+ \\
+ \\
+ \\
- \\
+
\end{array}\right]
$$

The last column $\mathcal{C}_{6}$ can be taken to be any 6 by 1 column having a + and a - entry, and no zeros. Let $\mathcal{C}=\left[\mathcal{C}_{5} \mid \mathcal{C}_{6}\right]$. In order to determine a matrix $[A \mid C] \in Q([\mathcal{S} \mid \mathcal{C}])$ with a positive (left) inverse $\left[\begin{array}{l}B_{1} \\ B_{2}\end{array}\right]$, the algorithm described in the proof of [2, Theorem 9.2.1] can be used. Then $B_{1}$ is a PLI of $A$, and the rows of $B_{2}$ are positive left nullvectors of $A$.

The next lemma is used to prove Theorem 2.10, in which square and nonsquare cases are combined. 
Lemma 2.9. Let $\mathcal{S}$ be an $m$ by $n$ sign pattern with $n \geq 2$ and let $\mathcal{T}$ be the sign pattern obtained from $\mathcal{S}$ by deleting the rows of zeros in $\mathcal{S}$. Then

(i) $\mathcal{S}$ is strong Hall if and only if $\mathcal{T}$ is strong Hall, and

(ii) $\mathcal{S}$ allows a positive (nonnegative) left inverse if and only if $\mathcal{T}$ allows a positive (nonnegative) left inverse.

Proof. Without loss of generality, assume that $\mathcal{S}=\left[\begin{array}{l}\mathcal{T} \\ O\end{array}\right]$. The proof of (i) follows immediately from the definition of strong Hall.

To prove (ii), suppose first that $\mathcal{S}$ allows a PLI. Let $A_{1} \in Q(\mathcal{T})$ and $A=\left[\begin{array}{c}A_{1} \\ O\end{array}\right] \in$ $Q(\mathcal{S})$ have $B=\left[\begin{array}{ll}B_{1} & B_{2}\end{array}\right]$ as a PLI. Then $B_{1} A_{1}=I_{n}$ and hence $\mathcal{T}$ allows a PLI. Next, suppose that $\mathcal{T}$ allows a PLI. Let $A_{1} \in Q(\mathcal{T})$ have $B_{1}$ as a PLI. With $J$ denoting the all 1's matrix, it is easily verified that $B=\left[\begin{array}{ll}B_{1} & J\end{array}\right]$ is a PLI for $A=\left[\begin{array}{c}A_{1} \\ O\end{array}\right] \in Q(\mathcal{S})$. Hence, $\mathcal{S}$ allows a PLI. The nonnegative case can be shown by a similar argument to that above.

Theorem 2.10. Let $m \geq n \geq 2$. The $m$ by $n$ sign pattern $\mathcal{S}$ allows a PLI if and only if

(i) each column of $\mathcal{S}$ has $a+$ and $a-$ entry;

(ii) $\mathcal{S}$ is strong Hall; and

(iii) the bipartite digraph $D\left(\mathcal{S}_{1}\right)$ of $\mathcal{S}_{1}$ is strongly connected whenever $\mathcal{S}$ is permutationally equivalent to $\left[\begin{array}{c}\mathcal{S}_{1} \\ O\end{array}\right]$ and $\mathcal{S}_{1}$ is an $n$ by $n$ sign pattern.

Proof. For the necessity, suppose that $\mathcal{S}$ allows a PLI. Then (i) and (ii) follow from Lemmas 2.2 and 2.3, and (iii) follows from Theorem 2.4 and Lemma 2.9 (ii).

For the sufficiency, first assume $m=n$. Then $\mathcal{S}$ is permutationally equivalent to $\mathcal{S}_{1}$, and by Theorem 2.4 the result follows from (ii) and (iii). Next, suppose that $m>n$. If $\mathcal{S}$ has no rows of zeros, then, by Theorem 2.7, the result follows from (i) and (ii). Otherwise, without loss of generality, assume that $\mathcal{S}=\left[\begin{array}{c}\mathcal{T} \\ O\end{array}\right]$, where $\mathcal{T}$ has no rows of zeros. By Lemma 2.9 (i), it follows from (ii) that $\mathcal{T}$ is strong Hall. Thus, if $\mathcal{T}$ is an $n$ by $n$ sign pattern, then (iii) and Theorem 2.4 imply that $\mathcal{T}$ allows a PLI. By Lemma 2.9 (ii), this implies that $\mathcal{S}$ allows a PLI. Otherwise, since it follows from (i) that each column of $\mathcal{T}$ has a + and a - entry, Theorem 2.7 implies that $\mathcal{T}$ allows a PLI. Therefore, by Lemma 2.9 (ii), $\mathcal{S}$ allows a PLI.

Remark 2.11. For $m \geq n \geq 2$, let $\mathcal{S}$ be an $m$ by $n$ sign pattern. Then the following hold:

(i) If $\mathcal{S}$ satisfies (i), (ii), and (iii) in Theorem 2.10, then so does every superpattern of $\mathcal{S}$. Hence, if $\mathcal{S}$ allows a PLI, then every superpattern of $\mathcal{S}$ allows a PLI.

(ii) Suppose that $\mathcal{S}=\left[\begin{array}{c}\mathcal{S}_{1} \\ O\end{array}\right]$, where $\mathcal{S}_{1}$ is a square sign pattern, satisfies (iii) in Theorem 2.10. Then, in contrast with Theorem 2.7 (i), there is no matrix $A=\left[\begin{array}{c}A_{1} \\ O\end{array}\right] \in Q(\mathcal{S})$ with a PLI that also has a positive left nullvector, since the equation $\left[\begin{array}{ll}y^{T} & z^{T}\end{array}\right]\left[\begin{array}{c}A_{1} \\ O\end{array}\right]=0$ and the fact that $A_{1}$ is nonsingular together imply that $y=0$.

The following theorem gives sufficient conditions for an $m$ by $n$ sign pattern with $m>n \geq 1$ to have a realization with a PLI and a positive left nullvector.

THEOREM 2.12. Let $\mathcal{S}$ be an $m$ by $n$ sign pattern with $m>n$ and let $\mathcal{T}$ be the $t$ by $n$ sign pattern obtained from $\mathcal{S}$ by deleting the rows of zeros in $\mathcal{S}$.

(i) If $n=1$ and $\mathcal{T}$ has $a+$ and $a-$ entry, then there exists a matrix in $Q(\mathcal{S})$ with a PLI and a positive left nullvector.

(ii) If $t>n \geq 2$ and $\mathcal{T}$ allows a PLI, then there exists a matrix in $Q(\mathcal{S})$ with a $P L I$ and a positive left nullvector.

Proof. (i) By Proposition 2.1, a + entry implies the existence of $A \in Q(\mathcal{S})$ with a 
PLI. Since $A$ has a positive and a negative entry, it can be easily verified that $A$ has a positive left nullvector.

(ii) When $m=t$, the result follows by Theorem 2.7. If $m>t>n$, then Theorem 2.7 implies that there exists a matrix $A \in Q(\mathcal{T})$ with a PLI $B$ and a positive left nullvector $y^{T}$. Note that the positive matrix $[B \mid J]$ is a PLI and the vector $\left[y^{T} 1 \cdots 1\right]$ is a positive left nullvector of the matrix $\left[\begin{array}{l}A \\ O\end{array}\right] \in Q(\mathcal{S})$. Hence, the result follows.

3. Nonnegative left inverses. In this section we determine structures of nonsquare sign patterns that allow an NLI, as well as structures of NLIs.

For $m \geq n$, let $\mathcal{S}$ be an $m$ by $n$ sign pattern with a realization of rank $n$. Then, by induction, it can be shown that $\mathcal{S}$ is permutationally equivalent to

$$
\left[\begin{array}{cccc}
\mathcal{S}_{11} & \mathcal{S}_{12} & \cdots & \mathcal{S}_{1 k} \\
O & \mathcal{S}_{22} & \cdots & \mathcal{S}_{2 k} \\
\vdots & & \ddots & \vdots \\
O & \cdots & O & \mathcal{S}_{k k}
\end{array}\right]
$$

where $k \geq 1, \mathcal{S}_{i i}$ is a square fully indecomposable sign pattern for each $i \in\{1, \ldots, k-$ $1\}$, and $\mathcal{S}_{k k}$ is strong Hall. Note that $\mathcal{S}$ is strong Hall if and only if $k=1$. If $\mathcal{S}$ is an $n$ by $n$ fully indecomposable sign pattern, then $\mathcal{S}$ allows a nonnegative (left) inverse if and only if $\mathcal{S}$ allows a positive (left) inverse; see [2, Theorems 9.2.1 and 9.2.3]. In addition, [2, Theorem 9.2.6] provides a complete characterization of $n$ by $n$ partly decomposable sign patterns that allow a nonnegative (left) inverse.

Remark 3.1. Suppose $m>n$. Let $\mathcal{S}^{\prime}$ be the square submatrix of $\mathcal{S}$ obtained by deleting the columns and rows associated with $\mathcal{S}_{k k}$. Suppose that $\mathcal{S}$ allows an NLI. Then the square sign pattern $\mathcal{S}^{\prime}$ also allows an NLI. Hence, for $k=2, \mathcal{S}^{\prime}$ is fully indecomposable and must satisfy one of the equivalent conditions in [2, Theorem 9.2.1] (see also Theorem 2.4), and for $k \geq 3, \mathcal{S}^{\prime}$ is partly decomposable and must satisfy the conditions in [2, Theorem 9.2.6]. Furthermore, by an argument similar to that in the proof of Lemma 2.3, it is easily verified that an NLI $B$ of a matrix in $Q(\mathcal{S})$ has the block form $B=\left[B_{i j}\right]$ with $1 \leq i, j \leq k$ and the $(i, j)$-block $B_{i j}=O$ whenever $i>j$. Thus, it follows that the strong Hall sign pattern $\mathcal{S}_{k k}$ also allows an NLI.

We now investigate various necessary and/or sufficient conditions for a strong Hall nonsquare sign pattern to allow an NLI. We first consider strong Hall sign patterns with $\mathrm{a}+$ and a - entry in each column.

Proposition 3.2. For $m>n \geq 2$, let $\mathcal{S}$ be an $m$ by $n$ strong Hall sign pattern with $a+$ and $a-$ entry in each column, and let $\mathcal{T}$ be the $t$ by $n$ sign pattern obtained from $\mathcal{S}$ by deleting the rows of zeros in $\mathcal{S}$. If $t>n$, then $\mathcal{S}$ allows an $N L I$. If $t=n$, then $\mathcal{S}$ allows an NLI if and only if $D(\mathcal{T})$ is strongly connected.

Proof. The result follows directly from Theorem 2.10 and the fact that if $\mathcal{S}$ allows a PLI, then $\mathcal{S}$ allows an NLI.

Let $\mathcal{I}_{n}$ denote the $n$ by $n$ sign pattern with $I_{n}$ as a realization, i.e., $I_{n} \in Q\left(\mathcal{I}_{n}\right)$. Clearly, $\mathcal{I}_{n}$ allows an NLI. Thus, in order to allow an NLI, an $m$ by $n$ sign pattern with $m \geq n$ need not have a - entry in each column as is required to allow a PLI (see Lemma 2.2), but clearly must have a + entry in each column. We first consider the case that $\mathcal{S}$ has a nonnegative column having only + or 0 entries. For ease of notation, we sometimes use $(M)_{i j}$ to denote the $(i, j)$-entry of a matrix $M$.

Proposition 3.3. For $m \geq n \geq 2$, let $\mathcal{S}$ be an $m$ by $n$ sign pattern with at least one nonnegative column. If $\mathcal{S}$ allows an $N L I$, then each nonnegative column has at most $m-n+1$ positive entries. 
Proof. Let $B$ be an NLI of $A \in Q(\mathcal{S})$, and let $t$ be the number of positive entries in any nonnegative column of $A$. Without loss of generality, assume that the first column of $A$ is a nonnegative column with its first $t$ entries positive. Since $(B A)_{h 1}=0$ for each $h \in\{2, \ldots, n\}$, it follows that $B$ has the block form $B=\left[B_{i j}\right]$ with $1 \leq i, j \leq 2$, where the $(2,1)$-block $B_{21}$ is the $(n-1)$ by $t$ zero matrix. Hence, the equality $\operatorname{rank} B=n$ implies that the rank of the $(n-1)$ by $(m-t)$ matrix $B_{22}$ is $n-1$. Thus, $n-1 \leq m-t$ and the result follows.

If all columns are nonnegative, then the following result gives a necessary and sufficient condition for such a sign pattern to allow an NLI.

TheOREM 3.4. For $m \geq n \geq 1$, let $\mathcal{S}$ be an $m$ by $n$ nonnegative sign pattern. Then $\mathcal{S}$ allows an NLI if and only if $\mathcal{S}$ is permutationally equivalent to

$$
\left[\begin{array}{c}
\mathcal{I}_{n} \\
\mathcal{T}
\end{array}\right]
$$

where $\mathcal{T}$ is an $(m-n)$ by $n$ nonnegative sign pattern.

Proof. The case $n=1$ follows directly from Proposition 2.1. Suppose that $n \geq 2$.

For the sufficiency, assume without loss of generality that

$$
\mathcal{S}=\left[\begin{array}{c}
\mathcal{I}_{n} \\
\mathcal{T}
\end{array}\right]
$$

Let $T \in Q(\mathcal{T})$ and $A=\left[\begin{array}{c}I_{n} \\ T\end{array}\right] \in Q(\mathcal{S})$. Since $\left[I_{n} \mid O\right] A=I_{n}$, it follows that $\mathcal{S}$ allows an NLI.

For the necessity, suppose that $\mathcal{S}=\left[s_{i j}\right]$ allows an NLI; i.e., there exist $A=$ $\left[a_{i j}\right] \in Q(\mathcal{S})$ and an $n$ by $m$ nonnegative matrix $B=\left[b_{i j}\right]$ such that $B A=I_{n}$. Let $i \in\{1, \ldots, n\}$. Since $(B A)_{i i}=1$, there exists $j_{i} \in\{1, \ldots, m\}$ such that $b_{i j_{i}} a_{j_{i} i}>0$. This implies that $s_{j_{i} i}=+$. Also, for each $k \in\{1, \ldots, n\} \backslash\{i\},(B A)_{i k}=0$ implies that $b_{i j_{i}} a_{j_{i} k}=0$. Thus, row $j_{i}$ of $\mathcal{S}$ is equal to row $i$ of $\mathcal{I}_{n}$. As this holds for each $i \in\{1, \ldots, n\}$, the result follows.

Remark 3.5. Let $\mathcal{S}=\left[\begin{array}{c}\mathcal{I}_{n} \\ \mathcal{J}\end{array}\right]$ be the $m$ by $n$ nonnegative sign pattern with $m \geq$ $n \geq 2$, where $\mathcal{J}$ is the sign pattern with all entries positive. Then, by Theorem $3.4, \mathcal{S}$ allows an NLI. However, in contrast with Remark 2.11 (i), Theorem 3.4 implies that no nonnegative superpattern of $\mathcal{S}$ (except $\mathcal{S}$ itself) allows an NLI.

Next, we consider sign patterns that have at least one nonnegative column and at least one column with $\mathrm{a}+$ and a - entry. We use $e_{i}$ to denote the $i$ th column vector of an identity matrix.

TheOREM 3.6. For $m \geq n \geq 2$, let $\mathcal{S}$ be an $m$ by $n$ sign pattern that has $p \geq 1$ nonnegative columns and $n-p \geq 1$ columns with $a+$ and $a-$ entry. Suppose that $\mathcal{S}$ allows an NLI. Then $\mathcal{S}$ is permutationally equivalent to a matrix of the form

$$
\left[\begin{array}{cc}
\mathcal{I}_{p} & \mathcal{S}_{12} \\
\mathcal{S}_{21} & \mathcal{S}_{22} \\
O & \mathcal{S}_{32}
\end{array}\right]
$$

where $\mathcal{S}_{21}$ is an $r$ by $p$ nonnegative sign pattern with no rows of zeros, $O$ is an $s$ by $p$ zero matrix with $s \geq 1$, and each of the last $n-p$ columns of $\mathcal{S}$ has $a+$ and $a-$ entry. Furthermore, if $\mathcal{S}$ is strong Hall, then $\mathcal{S}_{21}$ is not vacuous and has no column of zeros.

Proof. Without loss of generality, we may assume that the first $p$ columns of $\mathcal{S}$ are nonnegative, and that each of the last $n-p$ columns of $\mathcal{S}$ has a + and a - entry. 
Since $\mathcal{S}$ allows an NLI, so does the $m$ by $p$ nonnegative sign pattern consisting of the first $p$ columns of $S$. Therefore, by Theorem 3.4, we may permute the rows of $\mathcal{S}$ to obtain a matrix of the form (3.2), where $S_{21}$ is a nonnegative matrix with no row of zeros, $O$ is an $s$ by $p$ zero matrix with $s \geq 0$, and each of the last $n-p$ columns has a + and a - entry.

Let $A$ be a matrix in $Q(\mathcal{S})$ that has an NLI, say $B$. Since $B A=I_{n}$, each of the vectors $e_{1}^{T}, \ldots, e_{n}^{T}$ is a nontrivial, nonnegative linear combination of the rows of $A$. Since the first $p$ columns of $A$ are nonnegative and $n>p$, this requires that $s \geq 1$, and we conclude that $\mathcal{S}$ has the desired form.

If $\mathcal{S}_{21}$ is vacuous or has a column of zeros, then $\mathcal{S}$ has an $(m-1)$ by 1 zero submatrix. Hence $\mathcal{S}$ is not strong Hall, and the result follows by taking the contrapositive.

Proposition 3.7. For $m \geq n \geq 2$, let $\mathcal{S}$ be an $m$ by $n$ sign pattern that has $p \geq 1$ nonnegative columns and $n-p \geq 1$ columns with $a+$ and $a-$ entry. Suppose that $\mathcal{S}$ allows an NLI and has the form (3.2). Let

$$
A=\left[\begin{array}{cc}
A_{11} & A_{12} \\
A_{21} & A_{22} \\
O & A_{32}
\end{array}\right] \in Q(\mathcal{S})
$$

have an NLI $B=\left[\begin{array}{lll}B_{11} & B_{12} & B_{13} \\ B_{21} & B_{22} & B_{23}\end{array}\right]$, where each of $A_{21}, A_{22}, B_{12}$, and $B_{22}$ may be vacuous if $\mathcal{S}$ is not strong Hall. Then the following hold:

(i) $B_{11}$ is a diagonal matrix, and $B_{21}$ and $B_{22}$ are zero matrices.

(ii) The sign pattern $\mathcal{S}_{32}$ allows an NLI.

(iii) If row $q$ of $\mathcal{S}_{21}$ has at least two positive entries, then column $q$ of $B_{12}$ is a zero column.

(iv) Each column of $B_{12}$ has at most one positive entry. Furthermore, the sign pattern of $B_{12}$ is a subpattern of $\mathcal{S}_{21}^{T}$.

Proof. Assume that $\mathcal{S}_{21}$ is not vacuous.

Since $B A=I_{n}$, it follows that $B_{21} A_{11}+B_{22} A_{21}=O$. Moreover, since $B_{21}$, $B_{22}, A_{11}$, and $A_{21}$ are nonnegative, and no row of $A_{11}$ or $A_{21}$ is all zeros, $B_{21}=O$ and $B_{22}=O$. Also, $B A=I_{n}$ implies that $B_{11} A_{11}+B_{12} A_{21}=I_{p}$. Since $B_{11}, B_{12}$, $A_{11}$, and $A_{21}$ are nonnegative, both $B_{11} A_{11}$ and $B_{12} A_{21}$ are diagonal matrices. Since $A_{11} \in Q\left(\mathcal{I}_{p}\right), A_{11}$ is an invertible diagonal matrix, and hence $B_{11}$ is a diagonal matrix. Thus, (i) is proven.

Since $B_{21}$ and $B_{22}$ are zero matrices, and $B A=I_{n}, B_{23}$ is an NLI of $A_{32}$, and (ii) is proven.

Since $B_{12} A_{21}$ is a diagonal matrix and $B_{12}$ is nonnegative, the $i$ th row of $B_{12} A_{21}$ is a nonnegative linear combination of the rows of $A_{21}$ (weighted by the entries of the $i$ th row of $B_{12}$ ). As the $i$ th row of $B_{12} A_{21}$ is a nonnegative multiple of $e_{i}^{T}$, and $A_{21}$ is a nonnegative matrix with no row of zeros, it follows that if the $(i, j)$-entry of $B_{12}$ is nonzero, then the $j$ th row of $A_{21}$ is a multiple of $e_{i}^{T}$. In particular, this implies that each column of $B_{12}$ has at most one nonzero entry. If the $j$ th row of $A_{21}$ has at least two positive entries, then column $j$ of $B_{12}$ is a column of zeros, proving (iii). If the $(i, j)$-entry of $B_{12}$ is nonzero, then the $(j, i)$-entry of $A_{21}$ is nonzero, completing the proof of (iv).

If $\mathcal{S}_{21}$ is vacuous, then $A_{21}, A_{22}, B_{12}$, and $B_{22}$ are vacuous, in which case the proofs of (i) for $B_{11}, B_{21}$ and (ii) are similar, but statements (i) for $B_{22}$, (iii), and (iv) are vacuous. 
For $m \geq 2$, Proposition 3.2, Theorem 3.4, and the following theorem completely characterize the $m$ by 2 sign patterns that allow an NLI.

THEOREM 3.8. For $m \geq 2$, let $\mathcal{S}$ be an $m$ by 2 sign pattern such that the first column is nonnegative and the second column has $a+$ and $a-$ entry. Then $\mathcal{S}$ allows an NLI if and only if the first column of $\mathcal{S}$ has a + entry and $[0+]$ is a row of $\mathcal{S}$.

Proof. Suppose that $\mathcal{S}$ allows an NLI. Then the first column of $\mathcal{S}$ also allows an NLI. Hence, Theorem 3.4 implies that the first column of $\mathcal{S}$ has a + entry. By Theorem 3.6, we may assume without loss of generality that $\mathcal{S}$ is of the form (3.2). Since $\mathcal{S}_{32}$ is a column sign pattern, Propositions 3.7 (ii) and 2.1 imply that $\mathcal{S}_{32}$ has a + entry. Hence, $[0+]$ is a row of $\mathcal{S}$.

For the converse, suppose that the first column of $\mathcal{S}$ has a + entry and $[0+]$ is a row of $\mathcal{S}$. Suppose that $[+-]$ is also a row of $\mathcal{S}$. Then without loss of generality, $A \in \mathcal{S}$ has the form

$$
\left[\begin{array}{rr}
a & -b \\
u & v \\
0 & c
\end{array}\right]
$$

where $a, b, c>0$, and $u$ and $v$ are $(m-2)$ by 1 vectors. It is easy to verify that

$$
\left[\begin{array}{ccc}
1 / a & O & b / a c \\
0 & O & 1 / c
\end{array}\right]
$$

is an NLI of $A$.

Next suppose that $[+-]$ is not a row of $\mathcal{S}$. Then without loss of generality, $A \in \mathcal{S}$ has the form

$$
\left[\begin{array}{rr}
a & b \\
u & v \\
0 & -c \\
0 & d
\end{array}\right]
$$

where $a, c, d>0, b \geq 0$, and $u$ and $v$ are $(m-3)$ by 1 vectors. It is easy to verify that

$$
\left[\begin{array}{cccc}
1 / a & O & b / a c & 0 \\
0 & O & 1 / c & 2 / d
\end{array}\right]
$$

is an NLI of $A$.

Hence, $\mathcal{S}$ allows an NLI.

Note that the proof of Theorem 3.8 actually shows that if $\mathcal{S}$ is an $m$ by 2 matrix whose first column is nonnegative, second column has a + and a - entry, and $[0+]$ is one of its rows, then each matrix with sign pattern $\mathcal{S}$ has an NLI.

Example 3.9. The strong Hall sign pattern

$$
\mathcal{S}=\left[\begin{array}{cc}
+ & - \\
+ & - \\
0 & +
\end{array}\right]
$$

does not allow a PLI (by Lemma 2.2), but does allow an NLI (by Theorem 3.8) since

$$
\left[\begin{array}{lll}
1 & 0 & 1 / 2 \\
0 & 0 & 1 / 2
\end{array}\right]\left[\begin{array}{rr}
1 & -1 \\
1 & -1 \\
0 & 2
\end{array}\right]=I_{2}
$$


In general (as noted in the introduction) an NLI is not unique. For instance,

$$
\left[\begin{array}{ccc}
1 / 2 & 1 / 2 & 1 / 2 \\
0 & 0 & 1 / 2
\end{array}\right]
$$

is another NLI of the above matrix.

In the next theorem, it is shown that if a sign pattern $\mathcal{S}$ of the form (3.2) has a $(3,2)$-block $\mathcal{S}_{32}$ that allows an NLI or PLI, then some conditions on the negative entries in $\mathcal{S}_{12}$ insure that $\mathcal{S}$ allows an NLI.

TheOREM 3.10. For $m \geq n \geq 2$, let $\mathcal{S}$ be an $m$ by $n$ sign pattern of the form (3.2) with $p \geq 1, n-p \geq 1$, and $\mathcal{S}_{21}, \mathcal{S}_{22}$ arbitrary. Then the following hold:

(i) If $\mathcal{S}_{32}$ allows an NLI and $\mathcal{S}_{12}$ has only 0 or - entries, then $\mathcal{S}$ allows an NLI.

(ii) If $\mathcal{S}_{32}$ allows a PLI and each row of $\mathcal{S}_{12}$ has a - entry, then $\mathcal{S}$ allows an NLI.

Proof. (i) Let

$$
A=\left[\begin{array}{cc}
I_{p} & A_{12} \\
A_{21} & A_{22} \\
O & A_{32}
\end{array}\right] \in Q(\mathcal{S}),
$$

where $-A_{12} \geq 0$ and $A_{32}$ has $B_{23}$ as an NLI. Let

$$
B=\left[\begin{array}{lll}
I_{p} & O & B_{13} \\
O & O & B_{23}
\end{array}\right]
$$

with $B_{13}=-A_{12} B_{23}$, which is a nonnegative matrix. Then $B \geq 0, B A=I_{n}$, and hence the result follows.

(ii) Let $A \in Q(\mathcal{S})$ be of the form (3.3) and let $B$ be of the form (3.4). If $B_{23}$ is a PLI of $A_{32}$ and $B_{13}=-A_{12} B_{23}$, then $B_{13}>0$, provided that the negative entries of $A_{12}$ are sufficiently large in magnitude, and $B A=I_{n}$ as required.

4. Concluding remarks. In section 3 , we have characterized nonnegative sign patterns, strong Hall sign patterns with each column having a + and a - entry, and $m$ by 2 sign patterns that allow an NLI. For other cases, we have given some necessary or sufficient conditions for $\mathcal{S}$ to allow an NLI. A characterization for the blocks of the last column of a sign pattern $\mathcal{S}$ of the form (3.1) with $k \geq 2$ that allows an NLI remains open. We conclude by showing (in Theorem 4.2) that some conditions on the submatrix $\mathcal{S}_{k k}$ of a sign pattern $\mathcal{S}$ of the form (3.1) with $k \geq 2$ insure that $\mathcal{S}$ allows an NLI for arbitrary $\mathcal{S}_{1 k}, \ldots, \mathcal{S}_{k-1, k}$.

Let $\mathcal{S}$ allow a PLI and $A \in Q(\mathcal{S})$. The following proposition, which is used to prove Theorem 4.2, describes a relation between a PLI of $A$ and the qualitative behavior of solutions of $x^{T} A=b^{T}$. The latter equation is given in the introduction as motivation for studying PLIs and NLIs.

Proposition 4.1. For $m \geq n$, let $A$ be an $m$ by $n$ matrix. Then $A$ has a PLI if and only if for each $n$ by 1 nonzero vector $b \geq 0$ there exists an $m$ by 1 vector $x>0$ satisfying $x^{T} A=b^{T}$.

Proof. Suppose that an $n$ by $m$ matrix $B>0$ is a PLI of $A$. For an $n$ by 1 nonzero vector $b \geq 0$, it is clear that $\left(b^{T} B\right) A=b^{T}$ and $b^{T} B>0$. Hence, the result follows.

Next, suppose that for each $n$ by 1 nonzero vector $b \geq 0$ there exists an $m$ by 1 vector $x>0$ satisfying $x^{T} A=b^{T}$. Take $b$ to be the $i$ th column $e_{i}$ of $I_{n}$ and let $x_{i}>0$ 
be a solution of $x^{T} A=e_{i}^{T}$. Then the matrix

$$
B=\left[\begin{array}{c}
x_{1}^{T} \\
\vdots \\
x_{n}^{T}
\end{array}\right]
$$

is a PLI of $A$.

TheOREM 4.2. For $m>s \geq 1, n>t \geq 1$, and $m>n$, let $\mathcal{S}_{11}$ be an $s$ by $t$ sign pattern that allows an NLI and let $\mathcal{S}_{22}$ be an $(m-s)$ by $(n-t)$ sign pattern that allows a PLI. Suppose that if $n-t=1$, then $\mathcal{S}_{22}$ has a - entry, and if $n-t \geq 2$, then $\mathcal{S}_{22}$ is not permutationally equivalent to the sign pattern $\left[\begin{array}{l}\mathcal{T} \\ O\end{array}\right]$ in which $\mathcal{T}$ is a square sign pattern. Then, for an arbitrary $s$ by $(n-t)$ sign pattern $\mathcal{S}_{12}$, the sign pattern $\mathcal{S}=\left[\begin{array}{cc}\mathcal{S}_{11} & \mathcal{S}_{12} \\ O & \mathcal{S}_{22}\end{array}\right]$ allows an NLI.

Proof. Let $A_{11}$ be a matrix in $Q\left(\mathcal{S}_{11}\right)$ with $B_{11}$ as an NLI. By Theorem 2.12, there exists $A_{22} \in Q\left(\mathcal{S}_{22}\right)$ that has a PLI $B_{22}$ and a positive left nullvector $y^{T}$. Let $A_{12} \in Q\left(\mathcal{S}_{12}\right)$. Then $A_{12}$ can be written as $A_{12}=V_{1}-V_{2}$, where $V_{1}, V_{2} \geq 0$ and the entrywise (Hadamard) product $V_{1} \circ V_{2}=O$. Let $v_{i}^{T} \geq 0$ for $1 \leq i \leq s$ denote row $i$ of $V_{1}$. If $v_{i} \neq 0$, then by Proposition 4.1 there exists an $(m-s)$ by 1 vector $x_{i}>0$ such that $x_{i}^{T} A_{22}=v_{i}^{T}$. If $v_{i}=0$, then $x_{i}^{T} A_{22}=v_{i}^{T}=0$ when $x_{i}^{T}=y^{T}$. Thus, $K_{1}=\left[x_{1}, \ldots, x_{s}\right]^{T}>0$ and $K_{1} A_{22}=V_{1}$. Similarly, there exists $K_{2}>0$ such that $K_{2} A_{22}=V_{2}$.

Let $A_{12}(\epsilon)=\epsilon V_{1}-V_{2}=\left(\epsilon K_{1}-K_{2}\right) A_{22}$ for a sufficiently small $\epsilon>0$ such that $K_{2}-\epsilon K_{1}>0$. Note that $V_{1} \circ V_{2}=O$ implies that $A_{12}(\epsilon) \in Q\left(\mathcal{S}_{12}\right)$. Let $B_{12}=B_{11}\left(K_{2}-\epsilon K_{1}\right)$. Since $K_{2}-\epsilon K_{1}>0$ and $B_{11} \geq 0$ with no rows of zeros, it follows that $B_{12}>0$. It can be easily verified that $\left[\begin{array}{cc}B_{11} & B_{12} \\ O & B_{22}\end{array}\right]\left[\begin{array}{cc}A_{11} & A_{12}(\epsilon) \\ O & A_{22}\end{array}\right]=I_{n}$. Hence, the result follows.

Remark 4.3. Take $\mathcal{S}_{11}$ and $\mathcal{S}_{22}$ in Theorem 4.2 to be $\mathcal{S}^{\prime}$ in Remark 3.1 and $\mathcal{S}_{k k}$ in the form (3.1) with $k \geq 2$, respectively. Then the conditions on $\mathcal{S}_{k k}$ in Theorem 4.2 insure that the sign pattern $\mathcal{S}$ of the form (3.1) with $k \geq 2$ allows an NLI for arbitrary $\mathcal{S}_{1 k}, \ldots, \mathcal{S}_{k-1, k}$.

Acknowledgment. The authors thank the referee for comments that improved the readability of the paper.

\section{REFERENCES}

[1] A. Berman And B. D. Saunders, Matrices with zero line sums and maximal rank, Linear Algebra Appl., 40 (1981), pp. 229-235.

[2] R. A. Brualdi and B. L. Shader, Matrices of Sign-Solvable Linear Systems, Cambridge University Press, Cambridge, UK, 1995.

[3] R. A. Brualdi And B. L. Shader, Strong Hall matrices, SiAM J. Matrix Anal. Appl., 15 (1994), pp. 359-365.

[4] M. Fiedler And R. Grone, Characterizations of sign patterns of inverse positive matrices, Linear Algebra Appl., 40 (1981), pp. 237-245.

[5] T. Fujimoto And R. R. RAnade, Two characterizations of inverse-positive matrices: The Hawkins-Simon Condition and the Le Chatelier-Braun Principle, Electron. J. Linear Algebra, 11 (2004), pp. 59-65.

[6] C. R. Johnson, F. T. Leighton, and H. A. Robinson, Sign patterns of inverse positive matrices, Linear Algebra Appl., 24 (1979), pp. 75-83. 